TITLE:

\title{
A two-dimensional-reference interaction site model theory for solvation structure near solid-liquid interface.
}

\section{$\operatorname{AUTHOR(S):~}$}

lida, Kenji; Sato, Hirofumi

\section{CITATION:}

lida, Kenji ... [et al]. A two-dimensional-reference interaction site model theory for

solvation structure near solid-liquid interface.. The Journal of chemical physics 2011, 135(24): 244702.

ISSUE DATE:

2011-12-28

URL:

http://hdl.handle.net/2433/152421

RIGHT:

(C) 2011 American Institute of Physics 


\section{AIP $\begin{gathered}\text { mosoumalor } \\ \text { chemical Physics }\end{gathered}$}

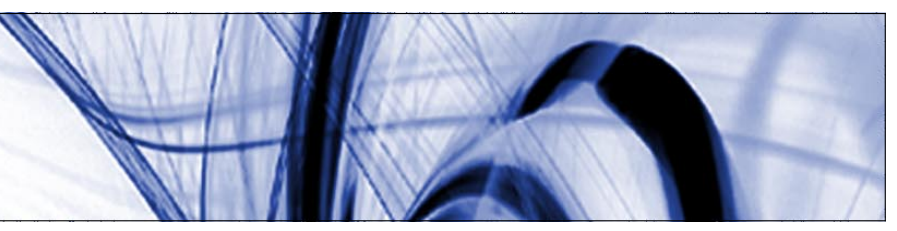

\section{A two-dimensional-reference interaction site model theory for solvation structure near solid-liquid interface}

Kenji lida and Hirofumi Sato

Citation: J. Chem. Phys. 135, 244702 (2011); doi: 10.1063/1.3668468

View online: http://dx.doi.org/10.1063/1.3668468

View Table of Contents: http://jcp.aip.org/resource/1/JCPSA6/v135/i24

Published by the American Institute of Physics.

\section{Related Articles}

Modeling the hydration of mono-atomic anions from the gas phase to the bulk phase: The case of the halide ions $\mathrm{F}-, \mathrm{Cl}-$, and $\mathrm{Br}-$

J. Chem. Phys. 136, 044509 (2012)

Structure, energetics, and reactions of alkali tetramers

J. Chem. Phys. 136, 014306 (2012)

Influence of solute-solvent coordination on the orientational relaxation of ion assemblies in polar solvents J. Chem. Phys. 136, 014501 (2012)

Theoretical study of the aqueous solvation of $\mathrm{HgCl}$ : Monte Carlo simulations using second-order Moller-Plessetderived flexible polarizable interaction potentials

J. Chem. Phys. 136, 014502 (2012)

On the solvation structure of dimethylsulfoxide/water around the phosphatidylcholine head group in solution JCP: BioChem. Phys. 5, $12 B 611$ (2011)

\section{Additional information on J. Chem. Phys.}

Journal Homepage: http://jcp.aip.org/

Journal Information: http://jcp.aip.org/about/about_the_journal

Top downloads: http://jcp.aip.org/features/most_downloaded

Information for Authors: http://jcp.aip.org/authors

\section{ADVERTISEMENT}

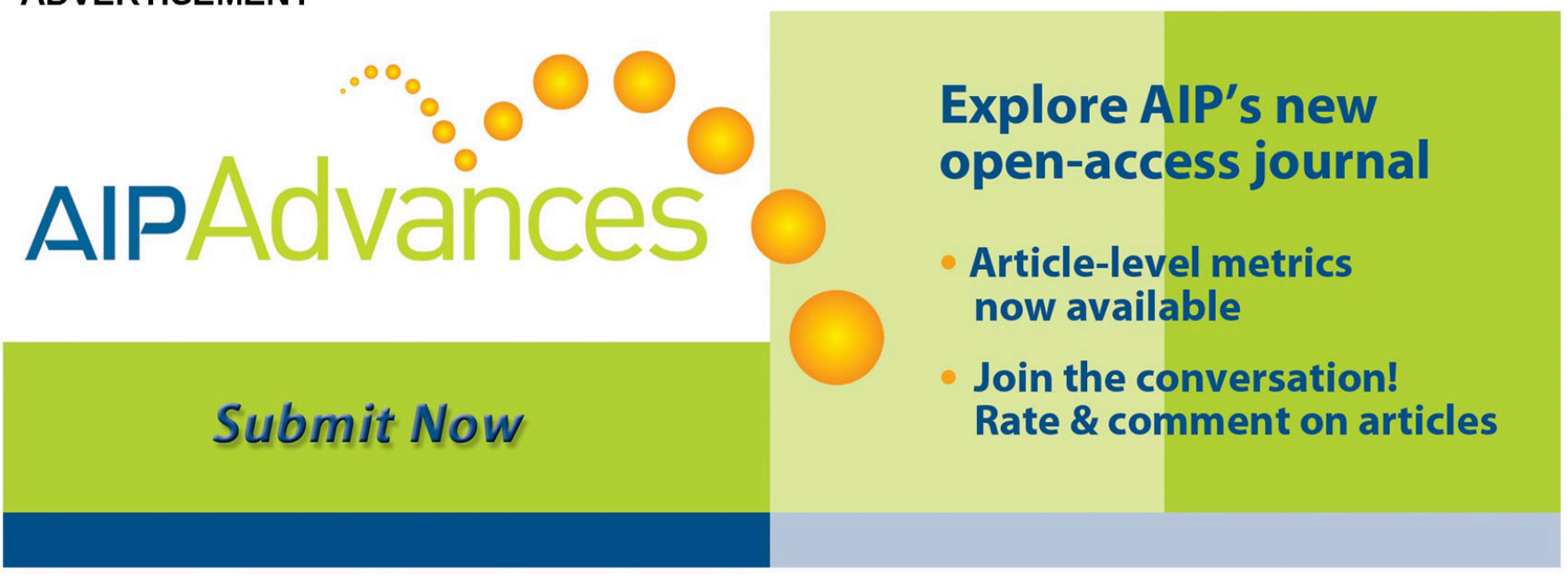




\title{
A two-dimensional-reference interaction site model theory for solvation structure near solid-liquid interface
}

\author{
Kenji lida and Hirofumi Sato ${ }^{a)}$ \\ Department of Molecular Engineering, Kyoto University, Kyoto 615-8510, Japan
}

(Received 29 August 2011; accepted 22 November 2011; published online 22 December 2011)

\begin{abstract}
We develop a new equation to describe solvation structure near solid-liquid interface at the atomiclevel. The developed equation focuses on anisotropy of solvation structure near the interface by using two-dimensional density distribution of solvent along two directions, one of which is perpendicular to the interface and the other is parallel to the interface. As a first application of the equation, we treat a system where a solid modeled by an atomistic wall is immersed in solvent water. The preferential adsorption position of water molecules and the change of water orientation by charging the wall are discussed. (C) 2011 American Institute of Physics. [doi:10.1063/1.3668468]
\end{abstract}

\section{INTRODUCTION}

Solvation near solid-liquid interface has been a subject of numerous studies, and recently, the molecular or atomic level information is gradually being clarified. ${ }^{1-6}$ For example, Fukuma et al. investigated mica-water interface with atomic force microscopy (AFM) and visualized water distribution near the interface. ${ }^{1,2}$ Schultz et al. investigated $\mathrm{Ag}(100)$-water interface with sum frequency generation (SFG) spectroscopy and showed that water orientation is dependent upon the applied electric potential. ${ }^{3}$

Theoretical or computational methods also provide valuable knowledge of the solvation structure near the interface, usually considering a solid as an atomistic wall. Molecular dynamics (MD) simulation is a representative and there are numerous studies of solid-liquid interface..$^{7-14}$ The atomiclevel knowledge that cannot be obtained with experimental methods is accumulated. The reference interaction site model (RISM) theory is the statistical mechanics for molecular liquids, and can be regarded as an alternative to MD simulation. The distinguishing feature of the RISM theory is to analytically treat an ensemble average of an infinite number of solvent molecules. ${ }^{15-19}$ The applicability of the theory is not limited to bulk liquid, and the theory has been extended to various solution systems. The polymer RISM theory treats the atomistic wall consisting of a virtually infinite number of atomic sites arranged in a periodic array. ${ }^{20-23}$ Akiyama and Hirata studied orientation of liquid water molecules near the wall with the polymer-RISM theory. ${ }^{24}$ Kovalenko et al. reported three-dimensional (3D) density-distribution of solvent near an atomistic wall with the 3D-RISM theory. ${ }^{17,25,26}$ Woelki et al. proposed the singlet-RISM theory where a solid is treated as an atomic site of infinite radius. ${ }^{27,28}$

In this study, we develop a new equation to describe solvation structure near solid-liquid interface at the atomiclevel. The new equation belongs to the RISM family and, thus, analytically treats an ensemble of infinite number of solvent molecules. The remarkable feature of the equation is

\footnotetext{
a) Author to whom correspondence should be addressed. FAX: +81-75-3832799. Electronic mail: hirofumi@moleng.kyoto-u.ac.jp.
}

focusing on the inherent feature near the interface, namely, anisotropy of solvation structure. The equation describes the anisotropic solvation structure as two-dimensional (2D) density distribution in a cylindrical coordinate system. The 2D distribution is along two directions, one of which is perpendicular to the interface and the other is parallel to the interface. The distribution along the perpendicular direction represents solvation shells near the interface. The distribution along the parallel direction is governed by atomistic features of the wall. The 2D-RISM equation is then combined with the polymer-RISM equation. The combined equation describes solvation structure around the wall consisting of atomic sites arranged in a 2D-periodic array. We apply the combined equation to the system in which an atomistic wall is immersed in solvent water. Solvation structure near the wall-water interface is discussed at the atomic-level. For example, adsorption position of water onto the wall and water orientation near the wall are discussed.

\section{THEORY}

\section{A. 2D-RISM equation}

Solvation structure is in general described with the six-dimensional Ornstein-Zernike (6D-OZ) equation. ${ }^{18,19}$ In this study, the system consists of an atomistic wall and a solvent, where the wall is fixed in the system. The 6D-OZ equation for this system is given as

$$
\begin{aligned}
& h\left(\mathbf{r}_{12}, \boldsymbol{\Omega}_{2}\right) \\
& \quad=c\left(\mathbf{r}_{12}, \mathbf{\Omega}_{2}\right)+n^{V}\left\langle\int d \mathbf{r}_{3} c\left(\mathbf{r}_{13}, \mathbf{\Omega}_{3}\right) h^{V}\left(\mathbf{r}_{32}, \mathbf{\Omega}_{3}, \mathbf{\Omega}_{2}\right)\right\rangle_{\mathbf{\Omega}_{3}},
\end{aligned}
$$

where 1 indicates the wall, 2 and 3 indicate the solvent molecules, respectively. $\mathbf{r}_{12}$ is defined as $\mathbf{r}_{12}=\mathbf{r}_{2}-\mathbf{r}_{1}$, where $\mathbf{r}_{1}$ is an arbitrary position in the wall and $\mathbf{r}_{2}$ is a center-of-mass of solvent molecule 2. In Eq. (1) and thereafter, we define $\mathbf{r}_{A B}$ as a $3 \mathrm{D}$ vector from a position of a particle $A\left(\mathbf{r}_{A}\right)$ to that of a particle $B\left(\mathbf{r}_{B}\right) . \boldsymbol{\Omega}_{A}$ is the orientation of a particle $A$ and \langle\rangle$_{\boldsymbol{\Omega}_{A}}$ denotes the averaging over $\boldsymbol{\Omega}_{A} . c$ is the direct correlation 


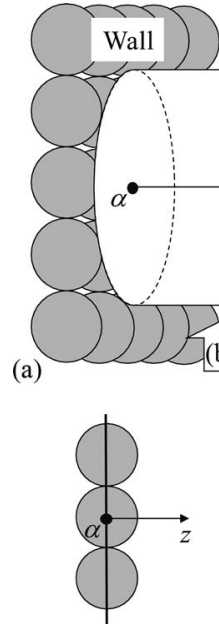

(b) (c)

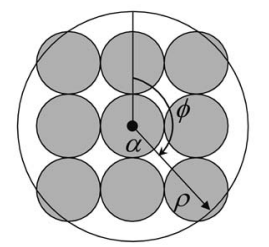

FIG. 1. (a) Cylindrical coordinate system. (b) View from the direction perpendicular to z-axis. (c) View from the direction parallel to z-axis.

function between the wall and solvent, and $h$ is the total correlation function between the wall and the solvent. The superscript $V$ denotes solvent, $n^{V}$ is the number density of solvent, and $h^{V}$ is the total correlation function of solvent. In Eq. (1), the orientation of the wall $\left(\boldsymbol{\Omega}_{1}\right)$ is omitted because here $\boldsymbol{\Omega}_{1}$ is fixed in the system. In the reciprocal space, $h$ is written as

$$
\begin{aligned}
h\left(\mathbf{k}, \boldsymbol{\Omega}_{2}\right) & =\int h\left(\mathbf{r}_{12}, \boldsymbol{\Omega}_{2}\right) e^{i \mathbf{k} \cdot \mathbf{r}_{12}} d \mathbf{r}_{12} \\
& =c\left(\mathbf{k}, \boldsymbol{\Omega}_{2}\right)+n^{V}\left\langle c\left(\mathbf{k}, \boldsymbol{\Omega}_{3}\right) h^{V}\left(\mathbf{k}, \boldsymbol{\Omega}_{3}, \boldsymbol{\Omega}_{2}\right)\right\rangle_{\mathbf{\Omega}_{3}} .
\end{aligned}
$$

To derive the equation for the $2 \mathrm{D}$ distribution from the $6 \mathrm{D}-\mathrm{OZ}$ equation, let us then introduce the cylindrical coordinate system shown in Fig. 1. An origin of the coordinate system is defined as a position of an arbitrary wall site $\alpha$, an axis perpendicular to the wall is defined as Z-axis, $\rho$ is the distance from the z-axis on a plane parallel to the wall, and $\phi$ is an angle along the wall. In this coordinate system, the position of a solvent site $\eta$ is defined as $\mathbf{r}_{\alpha \eta}=\left\{\rho_{\alpha \eta}, z_{\alpha \eta}, \phi_{\alpha \eta}\right\}$. The 2D total correlation function between the sites $\alpha$ and $\eta$, $h_{\alpha \eta}\left(\rho_{\alpha \eta}, z_{\alpha \eta}\right)$, can be given by averaging over $\phi_{\alpha \eta}$ and $\boldsymbol{\Omega}_{2}$ as

$$
\begin{aligned}
& h_{\alpha \eta}\left(\rho_{\alpha \eta}, z_{\alpha \eta}\right) \\
& \quad=\left\langle\int h\left(\mathbf{r}_{12}, \boldsymbol{\Omega}_{2}\right) \delta\left(\mathbf{r}_{12}-\mathbf{r}_{1 \alpha}-\mathbf{r}_{\eta 2}-\mathbf{r}_{\alpha \eta}\right) d \mathbf{r}_{12}\right\rangle_{\phi_{\alpha \eta}, \boldsymbol{\Omega}_{2}},
\end{aligned}
$$

where $\delta$ is the $\delta$-function, and the integral over $\mathbf{r}_{12}$ is to change the variable from $\mathbf{r}_{12}$ to $\mathbf{r}_{\alpha \eta} \cdot{ }^{17,18}$

Using Eqs. (2) and (3), $h_{\alpha \eta}$ in the reciprocal space is given as

$$
\begin{aligned}
& h_{\alpha \eta}\left(k^{\rho}, k^{z}\right) \\
& =\int h_{\alpha \eta}\left(\rho_{\alpha \eta}, z_{\alpha \eta}\right) e^{i \mathbf{k} \cdot \mathbf{r}_{\alpha \eta}} d \mathbf{r}_{\alpha \eta} \\
& =\left\langle\left. c\left(\mathbf{k}, \boldsymbol{\Omega}_{2}\right) e^{-i \mathbf{k} \cdot \mathbf{r}_{1 \alpha}} e^{-i \mathbf{k} \cdot \mathbf{r}^{2}{ }^{2}}\right|_{\phi_{\alpha \eta}, \boldsymbol{\Omega}_{2}}\right. \\
& +n^{V}\left\langle c\left(\mathbf{k}, \boldsymbol{\Omega}_{3}\right) h^{V}\left(\mathbf{k}, \boldsymbol{\Omega}_{3}, \boldsymbol{\Omega}_{2}\right) e^{-i \mathbf{k} \cdot \mathbf{r}_{1 \alpha}} e^{-i \mathbf{k} \cdot \mathbf{r}_{\eta} 2}\right\rangle_{\phi_{\alpha \eta}, \boldsymbol{\Omega}_{2}, \boldsymbol{\Omega}_{3}},
\end{aligned}
$$

where $k^{\rho}$ and $k^{z}$ are the $\rho$-component and $z$-component of $\mathbf{k}$, respectively. To perform the averaging, we then assume that the direct correlation function, $c$, can be written as the superposition of 2D site-site direct correlation functions, $\left\{\tilde{c}_{\alpha \eta}\right\}$, as follows:

$$
c\left(\mathbf{r}_{12}, \boldsymbol{\Omega}_{2}\right)=\sum_{\alpha \eta} \tilde{c}_{\alpha \eta}\left(\rho_{\alpha \eta}, z_{\alpha \eta}\right) .
$$

This assumption (Eq. (5)) is analogous to that employed to derive the RISM equation and the 3D-RISM equation. ${ }^{17,19,26}$ In the reciprocal space, Eq. (5) is written as

$$
\begin{aligned}
c\left(\mathbf{k}, \boldsymbol{\Omega}_{2}\right) & =\int c\left(\mathbf{r}_{12}, \boldsymbol{\Omega}_{2}\right) e^{i \mathbf{k} \cdot \mathbf{r}_{12}} d \mathbf{r}_{12} \\
& =\sum_{\alpha \eta} \tilde{c}_{\alpha \eta}\left(k^{\rho}, k^{z}\right) e^{i \mathbf{k} \cdot \mathbf{r}_{1 \alpha}} e^{i \mathbf{k} \cdot \mathbf{r}_{12}},
\end{aligned}
$$

where the integral over $\mathbf{r}_{12}$ is for fixed orientation of 1 and 2 , i.e., for the fixed intramolecular vectors $\left(\mathbf{r}_{1 \alpha}\right.$ and $\left.\mathbf{r}_{\eta 2}\right)$, and thus, the integral variable is transformed as $d \mathbf{r}_{12}=d \mathbf{r}_{\alpha \eta}$. This transformation is applied to derive the RISM equation. ${ }^{19}$

If the direct correlation function, $c$, in Eq. (4) is replaced with the superposition (Eq. (6)), then the following equation is obtained:

$$
\begin{aligned}
h_{\alpha \eta}\left(k^{\rho}, k^{z}\right)= & \sum_{\alpha^{\prime} \eta^{\prime}} e^{i \mathbf{k} \cdot \mathbf{r}_{\alpha \alpha^{\prime}}} \tilde{c}_{\alpha^{\prime} \eta^{\prime}}\left(k^{\rho}, k^{z}\right) \\
& \times\left[\omega_{\eta^{\prime} \eta}^{V}(|\mathbf{k}|)+n^{V} h_{\eta^{\prime} \eta}^{V}(|\mathbf{k}|)\right] .
\end{aligned}
$$

Here $\alpha^{\prime}$ and $\eta^{\prime}$ are wall and solvent sites, respectively, and $\left\langle e^{i \mathbf{k} \cdot \mathbf{r}_{\alpha \alpha^{\prime}}}\right\rangle_{\phi_{\alpha \eta}}=e^{i \mathbf{k} \cdot \mathbf{r}_{\alpha \alpha^{\prime}}}$ is applied because $e^{i \mathbf{k} \cdot \mathbf{r}_{\alpha \alpha^{\prime}}}$ is not dependent on $\phi_{\alpha \eta} . \omega_{\eta^{\prime} \eta}^{V}$ is the intramolecular correlation function of solvent. In the reciprocal space, $\omega_{\eta^{\prime} \eta}^{V}$ is given as

$$
\omega_{\eta^{\prime} \eta}^{V}(|\mathbf{k}|)=\frac{\sin \left(|\mathbf{k}|\left|\mathbf{r}_{\eta^{\prime} \eta}\right|\right)}{\left|\mathbf{k} \| \mathbf{r}_{\eta^{\prime} \eta}\right|}
$$

where $\mathbf{r}_{\eta^{\prime} \eta}$ is the vector from the site $\eta^{\prime}$ to the site $\eta$ in one solvent molecule. $h_{\eta^{\prime} \eta}^{V}$ in Eq. (7) is the site-site total correlation function of bulk solvent. Because of the isotropy of bulk solvent, $h_{\eta^{\prime} \eta}^{V}(\mathbf{k})$ can be reduced to $h_{\eta^{\prime} \eta}^{V}(|\mathbf{k}|)$. $\omega_{\eta^{\prime} \eta}^{V}$ and $h_{\eta^{\prime} \eta}^{V}$ have the same meanings as those used in the RISM equation. ${ }^{15-19}$

Because Eq. (7) includes $e^{i \mathbf{k} \cdot \mathbf{r}_{\alpha \alpha^{\prime}}} \quad\left(\mathbf{r}_{\alpha \alpha^{\prime}}=\left\{\rho_{\alpha \alpha^{\prime}}, z_{\alpha \alpha^{\prime}}\right.\right.$, $\left.\phi_{\alpha \alpha^{\prime}}\right\}$ ), the solution of Eq. (7) could be dependent on $\phi_{\alpha \alpha^{\prime}}$ besides $\rho_{\alpha \alpha^{\prime}}$ and $z_{\alpha \alpha^{\prime}}$. However, the solution in the real space, $h_{\alpha \eta}\left(\rho_{\alpha \eta}, z_{\alpha \eta}\right)$, is not dependent on $\phi_{\alpha \alpha^{\prime}}$. This is clearly seen by rewriting $e^{i \mathbf{k} \cdot \mathbf{r}_{\alpha \alpha^{\prime}}}$ using the Jacobi-Anger expansion,

$$
e^{i \mathbf{k} \cdot \mathbf{r}_{\alpha \alpha^{\prime}}}=e^{i k^{z} z_{\alpha \alpha^{\prime}}} \sum_{m=-\infty}^{\infty} i^{m} J_{m}\left(k^{\rho} \rho_{\alpha \alpha^{\prime}}\right) e^{i m \phi_{\alpha \alpha^{\prime}}},
$$

where $i$ is the imaginary unit, and $J_{m}$ is the $m$-th Bessel function. Applying Eq. (9), the terms of Eq. (7) other than the one corresponding to $m=0$ vanish by the inverse Fourier transformation because here $h_{\alpha \eta}$ and $\tilde{c}_{\alpha \eta}$ are not functions of $\phi$. That is to say, only the $m=0$ term gives a non-vanishing contribution in the real space. Equation (7) is, thus, rewritten to the equation named the 2D-RISM equation,

$$
\begin{aligned}
h_{\alpha \eta}\left(k^{\rho}, k^{z}\right)= & \sum_{\alpha^{\prime} \eta^{\prime}} \mathrm{w}_{\alpha \alpha^{\prime}}\left(k^{\rho}, k^{z}\right) \tilde{c}_{\alpha^{\prime} \eta^{\prime}}\left(k^{\rho}, k^{z}\right) \\
& \times\left[\omega_{\eta^{\prime} \eta}^{V}(|\mathbf{k}|)+n^{V} h_{\eta^{\prime} \eta}^{V}(|\mathbf{k}|)\right],
\end{aligned}
$$




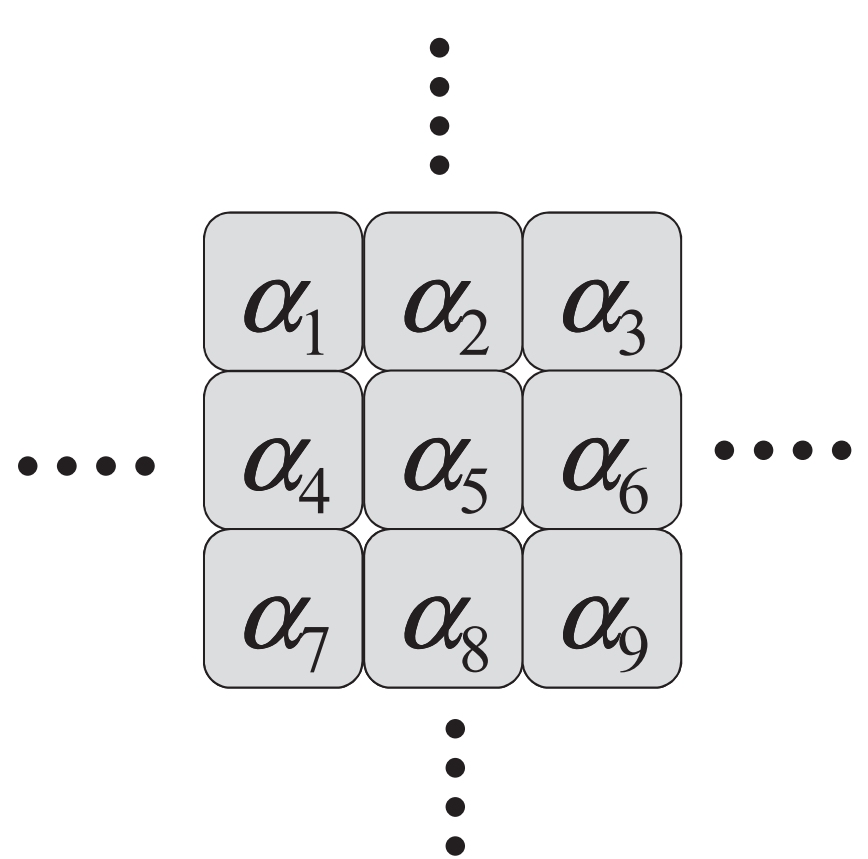

FIG. 2. Wall consisting of units arranged in a 2D periodic array.

where $\mathrm{w}_{\alpha \alpha^{\prime}}$ is the $2 \mathrm{D}$-intramolecular correlation function of the wall written as follows:

$$
\mathrm{w}_{\alpha \alpha^{\prime}}\left(k^{\rho}, k^{z}\right)=e^{i k^{z} z_{\alpha \alpha^{\prime}}} J_{0}\left(k^{\rho} \rho_{\alpha \alpha^{\prime}}\right) .
$$

$\mathrm{w}_{\alpha \alpha^{\prime}}$ describes the molecular structure of the wall.

\section{B. Combining with the polymer-RISM equation}

As is seen from Eq. (10), the equation to be solved is a matrix equation and the size of matrix becomes larger as the number of wall sites increases. It, thus, becomes cumbersome to obtain the solution of the matrix equation as the wall size increases. However, by combining with the polymer-RISM equation, ${ }^{20-24}$ we can drastically reduce the size of the matrix for a system, the wall of which is represented as shown in Fig. 2. The wall consists of identical units arranged in a 2D periodic array. Each unit labeled as $\boldsymbol{\alpha}_{i}(i=1, \ldots, N)$ is a finite set of atomic sites $\left\{\alpha_{i}, \alpha_{i}^{\prime}, \ldots, \alpha_{i}^{(M)}\right\}$. Using these notations, Eq. (10) is rewritten as follows:

$$
\begin{aligned}
h_{\alpha_{i} \eta}\left(k^{\rho}, k^{z}\right)= & \sum_{j}^{N} \sum_{\alpha^{\prime} \eta^{\prime}} \mathrm{w}_{\alpha_{i} \alpha_{j}^{\prime}}\left(k^{\rho}, k^{z}\right) \tilde{c}_{\alpha_{j}^{\prime} \eta^{\prime}}\left(k^{\rho}, k^{z}\right) \\
& \times\left[\omega_{\eta^{\prime} \eta}^{V}(|\mathbf{k}|)+n^{V} h_{\eta^{\prime} \eta}^{V}(|\mathbf{k}|)\right] .
\end{aligned}
$$

If the total number of unit $N$ is large enough, all units are virtually identical to each other, and thus,

$$
\begin{aligned}
h_{\alpha_{1} \eta}\left(k^{\rho}, k^{z}\right) & =h_{\alpha_{2} \eta}\left(k^{\rho}, k^{z}\right)=\cdots=h_{\alpha_{N} \eta}\left(k^{\rho}, k^{z}\right) \\
& =\frac{1}{N} \sum_{i}^{N} h_{\alpha_{i} \eta}\left(k^{\rho}, k^{z}\right) \equiv h_{\alpha \eta}\left(k^{\rho}, k^{z}\right) .
\end{aligned}
$$

Here, because of the identity, the index $i$ is dropped and $h_{\alpha_{i} \eta}\left(k^{\rho}, k^{z}\right)$ is rewritten as $h_{\alpha \eta}\left(k^{\rho}, k^{z}\right)$ in the last equation. Similarly, $\tilde{c}_{\alpha_{i} \eta}\left(k^{\rho}, k^{z}\right)$ is also rewritten as $\tilde{c}_{\alpha \eta}\left(k^{\rho}, k^{z}\right)$. Equa- tion (12) is then rewritten as

$$
\begin{aligned}
h_{\alpha \eta}\left(k^{\rho}, k^{z}\right)= & \sum_{\alpha^{\prime} \eta^{\prime}} \mathrm{W}_{\alpha \alpha^{\prime}}\left(k^{\rho}, k^{z}\right) \tilde{c}_{\alpha^{\prime} \eta^{\prime}}\left(k^{\rho}, k^{z}\right) \\
& \times\left[\omega_{\eta^{\prime} \eta}^{V}(|\mathbf{k}|)+n^{V} h_{\eta^{\prime} \eta}^{V}(|\mathbf{k}|)\right],
\end{aligned}
$$

where $\mathrm{W}_{\alpha \alpha^{\prime}}$ is the new intramolecular correlation function defined by

$$
\mathrm{W}_{\alpha \alpha^{\prime}}\left(k^{\rho}, k^{z}\right)=\frac{1}{N} \sum_{i j}^{N} \mathrm{w}_{\alpha_{i} \alpha_{j}^{\prime}}\left(k^{\rho}, k^{z}\right) .
$$

Now, according to Eq. (14), the size of the matrix depends on the number of sites in one unit $(M)$ and does not on $N$. The summation over the units is required only once to solve Eq. (15). Although the solution of Eq. (14) is dependent on $N$, the dependency is in practice negligibly small when $N$ is large enough as demonstrated in Sec. IV.

\section{Closure}

The two unknown functions, $\tilde{c}_{\alpha \eta}$ and $h_{\alpha \eta}$, appear in Eq. (14), and thus, another equation called closure relating these functions is needed. In this study, the following KHtype closure ${ }^{17,26}$ is adopted:

$$
\begin{aligned}
& \mathrm{g}_{\alpha \eta}\left(\rho_{\alpha \eta}, z_{\alpha \eta}\right) \\
& \quad= \begin{cases}\exp \left\{\chi_{\alpha \eta}\left(\rho_{\alpha \eta}, \mathrm{z}_{\alpha \eta}\right)\right\} & \left(\text { for } \quad \chi_{\alpha \eta}\left(\rho_{\alpha \eta}, z_{\alpha \eta}\right) \leq 0\right), \\
\chi_{\alpha \eta}+1 & \left(\text { for } \quad \chi_{\alpha \eta}\left(\rho_{\alpha \eta}, z_{\alpha \eta}\right)>0\right),\end{cases} \\
& \chi_{\alpha \eta}\left(\rho_{\alpha \eta}, z_{\alpha \eta}\right) \\
& \quad=-\beta u_{\alpha \eta}\left(\rho_{\alpha \eta}, z_{\alpha \eta}\right)+h_{\alpha \eta}\left(r_{\alpha \eta}, z_{\alpha \eta}\right)-\tilde{c}_{\alpha \eta}\left(\rho_{\alpha \eta}, z_{\alpha \eta}\right),
\end{aligned}
$$

where $\mathrm{g}_{\alpha \eta}\left(\rho_{\alpha \eta}, z_{\alpha \eta}\right)=h_{\alpha \eta}\left(\rho_{\alpha \eta}, z_{\alpha \eta}\right)+1$ is $2 \mathrm{D}$ pair correlation function (2D-PCF) between the wall site $\alpha$ and the solvent site $\eta . \beta=1 / k_{\mathrm{B}} T, k_{\mathrm{B}}$ is Boltzmann's constant. The interaction potential between $\alpha$ and $\eta, u_{\alpha \eta}$, is given as the sum of Coulombic and Lennard-Jones (LJ) terms as

$$
u_{\alpha \eta}\left(\rho_{\alpha \eta}, z_{\alpha \eta}\right)=4 \epsilon_{\alpha \eta}\left[\left(\frac{\sigma_{\alpha \eta}}{\left|\mathbf{r}_{\alpha \eta}\right|}\right)^{12}-\left(\frac{\sigma_{\alpha \eta}}{\left|\mathbf{r}_{\alpha \eta}\right|}\right)^{6}\right]+\frac{q_{\alpha} q_{\eta}}{\left|\mathbf{r}_{\alpha \eta}\right|},
$$

where $q_{\alpha}$ and $q_{\eta}$ are charges on $\alpha$ and $\eta$, respectively. $\sigma_{\alpha \eta}$ and $\epsilon_{\alpha \eta}$ are the Lennard-Jones parameters.

The procedure of the present theory is summarized as follows. $\omega_{\eta^{\prime} \eta}^{V}+n^{V} h_{\eta^{\prime} \eta}^{V}$ is first calculated with the RISM equation for bulk solvent. The intramolecular correlation function, $\mathrm{W}_{\alpha \alpha^{\prime}}$, is then calculated with Eq. (15). In the final step, $h_{\alpha \eta}$ and $\tilde{c}_{\alpha \eta}$ are iteratively calculated with Eqs. (14) and (16) until the convergence is achieved.

\section{COMPUTATIONAL DETAIL}

To solve the present equations, the 2D Fourier transform in the cylindrical coordinate system is required. The transform consists of the Hankel transform with respect to $\rho$ and the 1D Fourier transform with respect to $z$. The Hankel transform is performed with the logarithmic grids using Talman's algorithm. ${ }^{29}$ The number of grid points along $\rho$ is 512 and the 
grid spacing is $\Delta \ln \left(\rho / \rho_{0}\right)=0.02$, where $\rho_{0}$ is 1 bohr. The minimum of $\rho, \rho_{\min }$, is set as $\ln \left(\rho_{\min } / \rho_{0}\right)=-5.12$. The $1 \mathrm{D}$ Fourier transform is performed with the fast Fourier transform algorithm. The number of grid points along $z$ is 4096 and the spacing is $\Delta z=0.02$ bohr. To apply our theory to a charged wall, Ng's method ${ }^{30}$ is employed.

As the first application of the theory, we treat the model system where a single wall is immersed in aqueous solution. The atomic sites of the wall are arranged in accord with the face of a cubic lattice. The lattice constant $(a)$ is $1.5 \AA$ and the Lennard-Jones parameters of the atomic sites are $\sigma=1.500 \AA$ and $\epsilon=0.101 \mathrm{kcal} \mathrm{mol}^{-1}$. The lattice constant and the LJ parameters of the wall are the same as the system investigated by Crozier et al. using MD simulation, ${ }^{7}$ and are close to that by Woelki et al. using the singlet-RISM theory. ${ }^{27,28}$ For solvent water molecule, simple-point-charge-like model is employed (oxygen site: $\sigma_{\mathrm{O}}=3.166 \AA$ and $\epsilon_{\mathrm{O}}=0.155 \mathrm{kcal} \mathrm{mol}^{-1}$; hydrogen site: $\sigma_{\mathrm{H}}=1.000 \AA$ and $\left.\epsilon_{\mathrm{H}}=0.056 \mathrm{kcal} \mathrm{mol}^{-1}\right){ }^{31}$ Calculations are carried out at $298.15 \mathrm{~K}$ and the number density of solvent water of $n^{V}=0.033426 \AA^{-3}$.

In the following, we rewrite $\mathrm{g}_{\alpha \eta}\left(\rho_{\alpha \eta}, z_{\alpha \eta}\right)$ as $\mathrm{g}_{\mathrm{O}}(\rho, z)$ or $\mathrm{g}_{\mathrm{H}}(\rho, z)$ for simplicity, where $\mathrm{g}_{\mathrm{O}}(\rho, z)$ is $2 \mathrm{D}$-PCF between the wall site and the oxygen site and $\mathrm{g}_{\mathrm{H}}(\rho, z)$ is the $2 \mathrm{D}-\mathrm{PCF}$ between the wall site and the hydrogen site.

\section{RESULTS AND DISCUSSIONS}

Figure 3 shows the dependency on the total number of unit $N$, where $\mathrm{g}_{\mathrm{O}}(\rho=0.9 \AA, z=2.0 \AA)$ and $\mathrm{g}_{\mathrm{H}}(\rho=0.9 \AA$, $z=2.8 \AA$ ) are plotted along $N$. These positions correspond to peak tops in the case of $N=625\left(=25^{2}\right)$. The values of $\mathrm{g}_{\mathrm{O}}$ and $\mathrm{g}_{\mathrm{H}}$ are almost unchanged when $N$ is larger than $625 . N$ is, thus, fixed at 625 in the following discussion.

Figure 4(a) shows the contour map of $\mathrm{g}_{\mathrm{O}}(\rho, z)$. In the vicinity of $z=0 \AA$, any distinct peak is not found because the wall excludes solvent water from this area. The distribution increases with increasing distance from the wall $(z)$ and reaches the maximum at $z=2.0 \AA$. This area corresponds to the first solvation shell where water molecules are in contact with the wall. The maximum of $g_{0}$ is 3.8 , which is reasonably

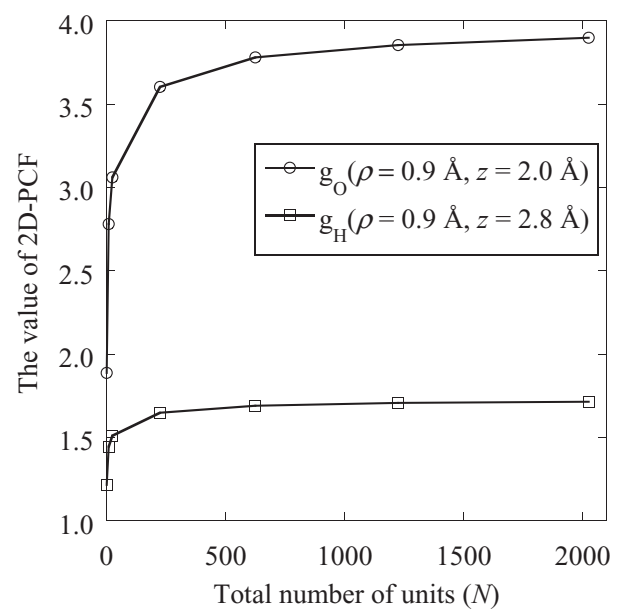

FIG. 3. Dependency on $N$ of $\mathrm{g}_{\mathrm{O}}(\rho=0.9 \AA, z=2.0 \AA)$ and $\mathrm{g}_{\mathrm{H}}(\rho=0.9 \AA$, $z=2.8 \AA$ ) .
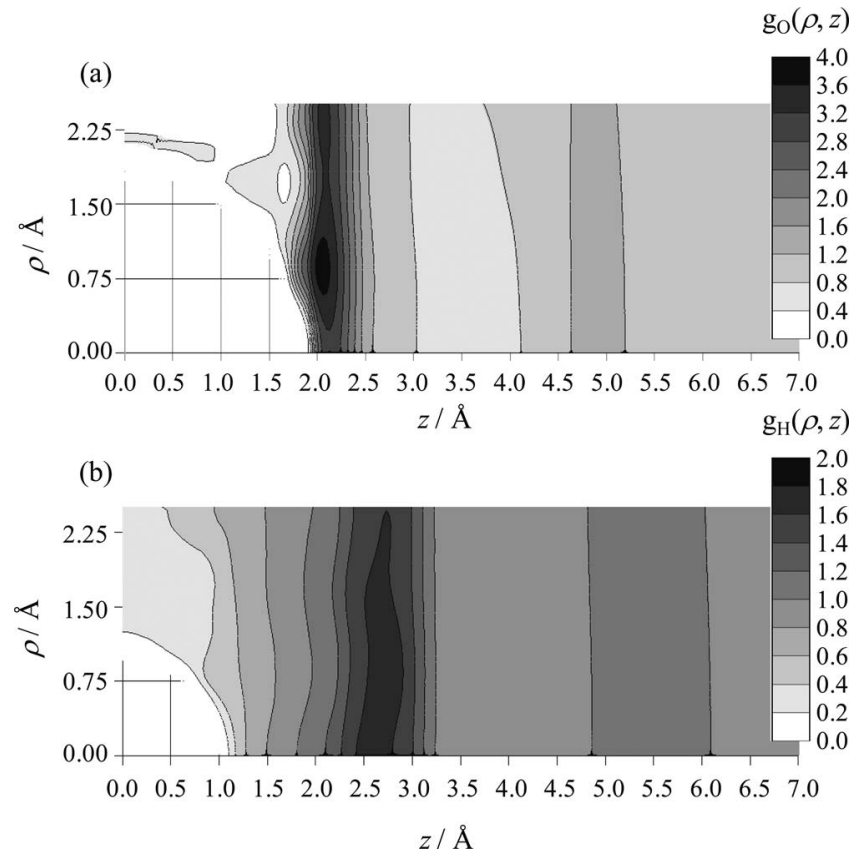

FIG. 4. Contour map of 2D-PCF between the wall site and the oxygen site (a), and the hydrogen site (b). The wall is neutral.

agree with the previous studies employing analogous model system: 3.2 (singlet-RISM) (Ref. 28) and 4.0 (MD). ${ }^{7}$ Note that the distributions of the previous studies are averaged over $\rho$, namely, the definition of $\mathrm{g}_{\mathrm{O}}$ is slightly different from that in the present study where the dependency on $\rho$ is also illustrated. The small distribution around $z=0.0 \AA \sim 1.0 \AA$ and $\rho=2.25 \AA$ seems to be an artifact, probably caused by the adopted closure that affects the description of the short-range region. ${ }^{32}$ Further careful investigations would be necessary to confirm it, but it is noted that the present theory yields the first solvation shell that reasonably agrees with the previous studies, as is described in the following discussion.

$\mathrm{g}_{\mathrm{O}}$ at $z \sim 2$ is especially dependent on $\rho$. The maximum of $\mathrm{g}_{\mathrm{O}}$ is at $\rho=0.9 \AA, z=2.0 \AA$, and the distribution expands from $\rho=0.6 \AA$ to $1.2 \AA$, where $\mathrm{g}_{\mathrm{O}}$ is larger than 3.6. Figure 5 illustrates this area, which includes bridge positions $(a / 2=0.75 \AA)$ and hollow positions $(a / \sqrt{2}=1.06 \AA)$. It is also noted that the height of $\mathrm{g}_{\mathrm{O}}$ at the on-top position ( $\rho=0.0 \AA, z=2.0 \AA$ ) is lower by about 0.7 compared to

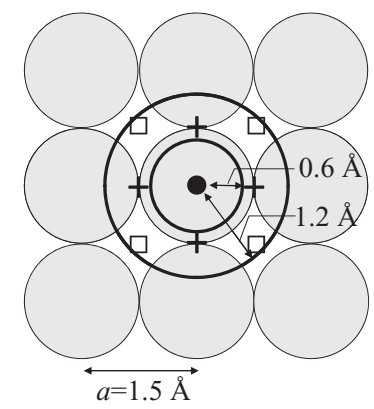

FIG. 5. Relationship between the area from $\rho=0.6 \AA$ to $1.2 \AA$ and the molecular structure of the wall. $\bullet$ : on-top position $(\rho=0 \AA)$; + : bridge position $(\rho=a / 2) ; \square$ : hollow position $(\rho=a / \sqrt{2})$. 


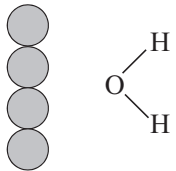

(a)

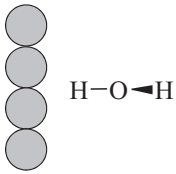

(b)
FIG. 6. (a) and (b) Orientation of water molecule near the wall.

the maximum. In other words, water molecules are preferably adsorbed on bridge and/or hollow positions compared to ontop positions.

We found another maximum at $z \sim 5 \AA$, corresponding to the second solvation shell. Different from the first solvation shell $(z \sim 2 \AA)$, the profile of the second solvation shell is virtually independent of $\rho$, indicating that the effect of atomistic feature of the wall is negligibly small at this area.

Figure 4(b) shows the contour map of $\mathrm{g}_{\mathrm{H}}(\rho, z)$. The distinct peak is found in the area from $z=2.5 \AA$ to $3.0 \AA$, which is slightly distant from the first solvation shell of the oxygen site $(z \sim 2 \AA)$. $\mathrm{g}_{\mathrm{O}}(\rho, z)$ and $\mathrm{g}_{\mathrm{H}}(\rho, z)$, therefore, show that the hydrogen site tends to be located further from the wall than the corresponding oxygen site as depicted in Fig. 6(a).

The solvation structures change by charging the wall. Figure 7(a) shows the contour map of $\mathrm{g}_{\mathrm{O}}(\rho, z)$, where all atomic sites of the wall are charged to $-0.04213|e|$. The applied charges correspond to $-0.3 \mathrm{C} \cdot \mathrm{m}^{-2}$. This surface charge density is chosen to be the same as the previous works. ${ }^{7,28}$ The profile of $g_{O}$ looks similar to that around the neutral wall at a glance. However, the peak top at $\rho=0.9 \AA, z=2.0 \AA$ slightly increases from 3.8 (neutral) to 4.0 (charged). This increase may be interesting because the oxygen site is more preferably adsorbed on the negatively charged wall in spite of the electrostatic repulsion between the oxygen site and the wall.
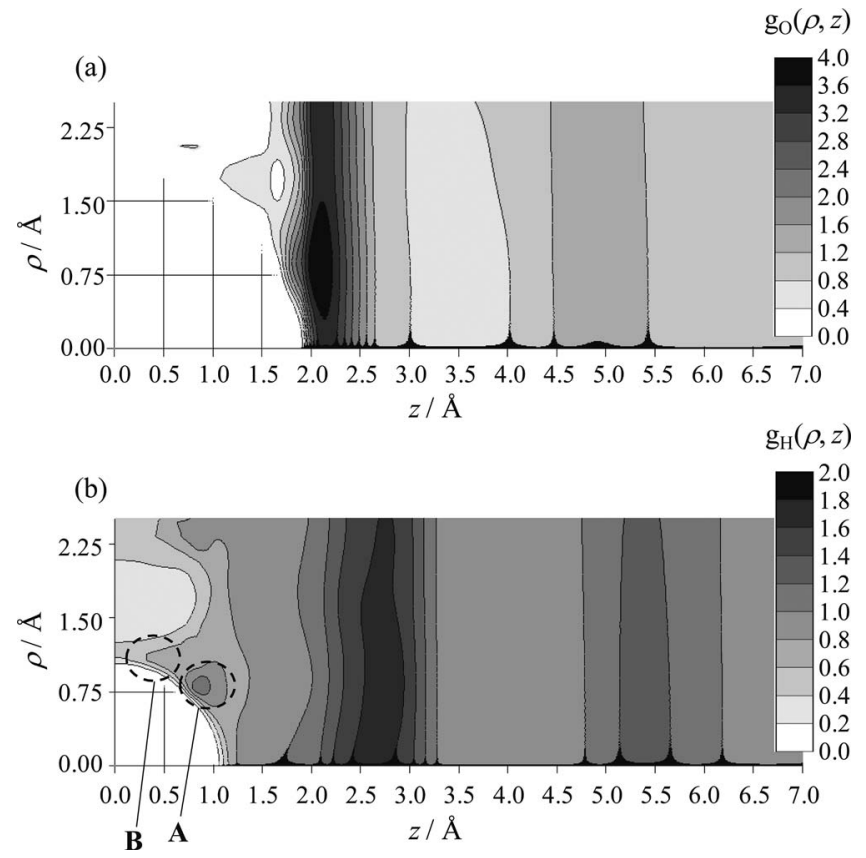

FIG. 7. Contour map of 2D-PCF between the wall site and the oxygen site (a), and the hydrogen site (b). The wall is negatively charged.
The increase is attributed to strong attractive interaction between the hydrogen site and the wall. Figure 7(b) shows the contour map of $\mathrm{g}_{\mathrm{H}}(\rho, z)$ near the negatively charged wall. The probability becomes slightly higher especially near $\mathbf{B}$ to form a ridge, and a distinct peak is found at $\mathbf{A}$. Based on the geometrical consideration, $\mathbf{A}$ includes bridge positions and B includes hollow positions, respectively (cf. Fig. 5). These changes are consistent with the previous works. ${ }^{7,28}$ As illustrated in Fig. 6(a), the distribution, in which two hydrogen atoms are further than the oxygen atom, is dominative before the charging. The aforementioned changes in $\mathbf{A}$ and $\mathbf{B}$ indicate that a mixing ratio of another configuration displayed in Fig. 6(b) becomes greater, namely, one of $\mathrm{O}-\mathrm{H}$ bond is directed perpendicular to the wall. The attractive interaction between the hydrogen site and the negatively charged wall is consistent with the above-mentioned increase of $\mathrm{g}_{\mathrm{O}}$ in the area of the first solvation shell.

\section{CONCLUSION}

In this study, we developed the 2D-RISM equation for solvation structure near solid-liquid interface. The developed equation focuses on the anisotropy of solvation structure near the interface by using the 2D density distribution in the cylindrical coordinate system. The 2D-RISM equation was then combined with the polymer-RISM equation to treat the solvation near the wall consisting of atomic sites in a 2D-periodic array. The model system was studied as the first application, and we found that water molecules in the first solvation shell are preferably adsorbed on hollow and/or bridge positions than on-top positions, whereas those in the second solvation shell do not show any specific preference. A new peak appears in the first solvation shell upon charging the wall, indicating that the contribution from another orientational configuration also becomes visible by the applied electric field. This change is assigned as follows: $\mathrm{O}-\mathrm{H}$ bond is directed perpendicular to the negatively charged wall. The interaction between the hydrogen site and the negatively charged wall is consistent with the increase of $g_{O}$.

\section{ACKNOWLEDGMENTS}

This work has been financially supported by the Grant-in Aid (20550013 and 22018016). K.I. thanks the Grand-in Aid for JSPS Fellows. The authors were supported by the Ministry of Education, Culture, Sports, Science and Technology (MEXT) Japan.

${ }^{1}$ T. Fukuma, K. Kobayashi, K. Matsushige, and H. Yamada, Appl. Phys. Lett. 87, 034101 (2005).

${ }^{2}$ T. Fukuma, Sci. Technol. Adv. Mater. 11, 033003 (2010).

${ }^{3}$ Z. D. Schultz, S. K. Shaw, and A. A. Gewirth, J. Am. Chem. Soc. 127, 15916 (2005).

${ }^{4}$ H. Noguchi, T. Okada, and K. Uosaki, Faraday Discuss. 140, 125 (2008).

${ }^{5}$ M. F. Toney, J. N. Howard, J. Richer, G. L. Borges, J. G. Gordon, O. R. Melroy, D. G. Wiesler, D. Yee, and L. B. Sorensen, Nature (London) 368, 444 (1994).

${ }^{6}$ M. F. Toney, J. N. Howard, J. Richer, G. L. Borges, J. G. Gordon, O. R. Melroy, D. G. Wiesler, D. Yee, and L. B. Sorensen, Surf. Sci. 335, 326 (1995). 
${ }^{7}$ P. S. Crozier, R. L. Rowley, and D. Henderson, J. Chem. Phys. 113, 9202 (2000).

${ }^{8}$ P. S. Crozier, R. L. Rowley, and D. Henderson, J. Chem. Phys. 114, 7513 (2001).

${ }^{9}$ D. A. Rose and I. Benjamin, J. Chem. Phys. 95, 6856 (1991).

${ }^{10}$ S. K. Reed, O. J. Lanning, and P. A. Madden, J. Chem. Phys. 126, 084704 (2007).

${ }^{11}$ E. Spohr, J. Phys. Chem. 93, 6171 (1989).

${ }^{12}$ E. Spohr and K. Heinzinger, Chem. Phys. Lett. 123, 218 (1986).

${ }^{13}$ G. D. Smith, O. Borodin, S. P. Russo, R. J. Rees, and A. F. Hollenkamp, Phys. Chem. Chem. Phys. 11, 9884 (2009).

${ }^{14}$ P. Zarzycki, S. Kerisit, and K. M. Rosso, J. Phys. Chem. C 114, 8905 (2010).

${ }^{15}$ D. Chandler and H. C. Andersen, J. Chem. Phys. 57, 1930 (1972).

${ }^{16}$ F. Hirata and P. J. Rossky, Chem. Phys. Lett. 83, 329 (1981).

${ }^{17}$ Molecular Theory of Solvation, edited by F. Hirata (Kluwer, Dordrecht, The Netherlands, 2003).

${ }^{18}$ J.-P. Hansen and I. R. McDonald, Theory of Simple Liquids, 3rd ed. (Academic, London, 2006).
${ }^{19}$ C. G. Gray and K. E. Gubbins, Theory of Molecular Fluids (Oxford University Press, New York, 1984).

${ }^{20}$ D. Chandler, Y. Singh, and D. M. Richardson, J. Chem. Phys. 81, 1975 (1984).

${ }^{21}$ K. S. Schweizer and J. G. Curro, Phys. Rev. Lett. 58, 246 (1987).

${ }^{22}$ J. G. Curro and K. S. Schweizer, J. Chem. Phys. 87, 1842 (1987).

${ }^{23}$ F. Hirata and R. M. Levy, Chem. Phys. Lett. 136, 267 (1987).

${ }^{24}$ R. Akiyama and F. Hirata, J. Chem. Phys. 108, 4904 (1998).

${ }^{25}$ A. Kovalenko and F. Hirata, Chem. Phys. Lett. 290, 237 (1998).

${ }^{26}$ A. Kovalenko and F. Hirata, J. Chem. Phys. 110, 10095 (1999).

${ }^{27}$ S. Woelki, H.-H. Kohler, and H. Krienke, J. Phys. Chem. B 111, 13386 (2007).

${ }^{28}$ S. Woelki, H.-H. Kohler, and H. Krienke, J. Phys. Chem. B 112, 3365 (2008).

29 J. D. Talman, J. Comput. Phys. 29, 35 (1978).

${ }^{30}$ K.-C. Ng, J. Chem. Phys. 61, 2680 (1974).

${ }^{31}$ H. J. C. Berendsen, J. P. M. Postma, W. F. van Gunsteren, and J. Hermans, in Intermolecular Forces, edited by B. Pullman (Reidel, Dordrecht, 1981).

${ }^{32}$ A. Kovalenko and F. Hirata, J. Chem. Phys. 133, 2793 (2000). 